\title{
Preoperative Optimization of Modifiable Risk Factors Affect Out- come in Complex Ventral Hernia Repair
}

\author{
Jai P. Singh* \\ Department of Surgery, Oswego Hospital, USA
}

"Corresponding author: Jai P. Singh, Department of Surgery, Oswego Hospital, 110 W 7th Street, Oswego, New York, USA. Tel: +13153426771; Fax:+13153422783, Email: drjp04@gmail.com

Citation: Singh JP (2018) Preoperative Optimization of Modifiable Risk Factors Affect Outcome in Complex Ventral Hernia Repair. J Surg: JSUR-1118. DOI: 10.29011/2575-9760. 001118

Received Date: 10 March, 2018; Accepted Date: 13 March, 2018; Published Date: 20 March, 2018

\begin{abstract}
Ventral hernia repair is one of the most commonly performed procedures worldwide. Hernia recurrence along with other complications of ventral hernia repair adversely affect patients and have substantial socio-economic impact. Identifying factors, which could affect postoperative outcome, is therefore very important. Factors like smoking, diabetes, history of MRSA infection, obesity and poor nutrition adversely affect wound healing process and lead to increased risk of infection and recurrence. Preoperative optimization of modifiable risk factors has shown to improve the outcome in terms of decreased wound complications and hernia recurrence
\end{abstract}

Keywords: Arginine; Diabetes; Infection; MRSA Decontamination; Nutrition; Obesity; Recurrence; Smoking; Ventral Hernia; Wound Healing

\section{Abbreviations}

$\begin{array}{llc}\text { SSI } & : & \text { Surgical Site Infection } \\ \text { SSO } & : & \text { Surgical Site Occurrence } \\ \text { MRSA } & : & \text { Methicillin Resistant Staphylococcus aureus } \\ \text { iNOS } & : & \text { Inducible Nitric Oxide Synthase } \\ \text { ASE1 } & : & \text { Arginase } \\ \text { OR } & : & \text { Odds Ratio } \\ \text { BMI } & : & \text { Body Mass Index }\end{array}$

\section{Introduction}

Ventral hernia repair is one of the most commonly performed surgical procedures worldwide. There are more than 350,000 ventral hernias repaired in the United States annually with $25 \%$ of them being complex hernia repair. Nearly one third of all individuals undergo some form of laparotomy in their life time and around $28 \%$ of them end up having incisional hernia [1]. Recurrence rate after ventral hernia repair is around $24-43 \%$. Hernia recurrence along with other complications of ventral hernia repair adversely affect patients and have substantial socio-economic impact [1]. Identifying factors, which could affect postoperative outcome, is therefore very important. Many factors have been identified including smoking, obesity, nutritional status, history of MRSA infection and diabetes, which are considered to be modifiable risk factors.

\section{Understanding Wound Healing}

Wound healing is a dynamic process consisting of four continuous, overlapping, and precisely programmed phases [2]. The four phases of wound healing are hemostasis, inflammatory phase, proliferation and remodeling [2]. Wound healing starts with hemostasis which includes vasoconstriction, activation of platelets and coagulation cascade leading to hemostatic clot formation, which is predominantly composed of polymerized fibrin crosslinked fibronectin $[2,3]$. Inflammatory phase then follows with accumulation of neutrophils and fibroblasts in the wound cavity. Neutrophils control bacterial contamination by phagocytosis and initiate tissue debridement by releasing proteolytic enzymes. The wound is then sequestered by monocytes as number of neutrophils diminishes. Monocytes transform into macrophages, which then facilitate continued debridement and eradication of microbes [3]. Macrophages release a range of cytokines such as interleukin-1 (IL-1) and tissue necrosis factor- $\alpha$ (TNF- $\alpha$ ) and growth factors such as vascular endothelial growth factor and 


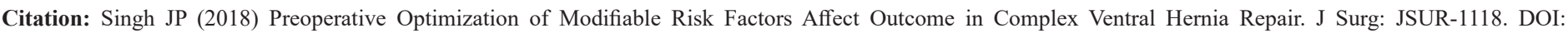
$10.29011 / 2575-9760.001118$

TGF- $\beta 1$ to attract reparative cells and orchestrate subsequent healing Events [3]. A concurrent process to inflammatory process is wound contraction, which is facilitated by transformation of fibroblasts into myofibroblats [3]. Proliferation process then starts with upregulation of reparative cells 3-4 days after the wounding. Fibroblasts secrete extracellular matrix components and collagen; epithelial and epidermal cells regenerate the barrier to the exterior; and the endothelial cells form new vessels [3]. After 2 to 3 weeks, remodeling replaces proliferation as the collagen fibrils undergo gradual modification in an ongoing process of synthesis and degradation. Covalent bonds are formed between the collagen molecules and fibrils become aligned along the tension lines, a process that continues with a gradually reducing intensity, for months to years after the initial surgical trauma [3].

\section{Effect of Smoking on Wound Healing}

Cigarette smoking has been found to induce vasoconstriction due to higher sympathetic tone caused by catecholamine release resulting in reduced subcutaneous blood flow by up to $40 \%$ [4,5]. The tissue oxygen tension is decreased by up to $27 \%[4,6]$ causing prolonged tissue acidosis that increases the risk of infection and tissue necrosis [3]. Tobacco smoke contains various compounds including nicotine, carbon monoxide, hydrogen cyanide, nitrogen oxides, N-nitrosamines, aldehydes, and polyaromatic hydrocarbons [3]. A study by Sorensen et al showed that the cutaneous and subcutaneous blood flows were affected differently by nicotine and smoking. Intravenous nicotine infusion increased tissue oxygen tension, whereas smoking caused significant reduction in tissue oxygen tension, blood flow and aerobic metabolism [4]. These findings suggest that the smoke constituents other than nicotine contribute to the vasoactive effect of smoking.

The formation of hemostatic clot is enhanced by smoking as a result of platelet activation and blood fibrinogen release [3]. The wound infiltration of inflammatory cells including both neutrophils and monocyte-macrophage is attenuated in smokers. Smoking alters the function of inflammatory cells as well leading to connective tissue degradation due to excessive protease release and reduced protease inhibition [3]. Studies have shown that phagocytic activity of neutrophils and macrophages is significantly impaired by cigarette smoke extract leading to reduced ability to control bacterial wound infection [7-9]. Wound contraction is also enhanced in smokers, which causes premature wound closure leading to increased susceptibility to infection [3]. Smoking releases a variety of reactive oxygen radicals, which have various detrimental effects on inflammatory cell function including impairment of neutrophil and monocyte migration [10], attenuation of oxidative bactericidal activity, which is their primary bactericidal mechanism [7,9], excessive release of proteolytic enzymes [11] and inactivation of protease inhibitors [12]. The surgical trauma reduces tissue oxygen as the blood supply is cut off and the diffusion distance is increased by hemostatic wound clot [13]. Hypoxia promotes bacterial colonization, which multiply by consuming glucose and oxygen. The already reduced oxygen tension in smokers increases the susceptibility to postsurgical infection by impairing oxidative killing of bacteria by neutrophils and macrophages [3].

Smoking affects the proliferative and remodeling phase of healing by inhibition of fibroblast chemotaxis, migration and proliferation in addition to reduced collagen III and I production [3]. The reduced collagen deposition causes impairment of wound angiogenesis as growing vessels are dependent on collagen for physical support [3]. Formation of new epithelium and epidermis is affected by smoking as cellular epidermis and stratum corneum have been found to be thinner in smokers [3]. Smokers are found to have low level of Vitamin $\mathrm{C}$ as it acts as a potent antioxidant to reduce oxidative stress from smoking through scavenging and binding of metal ions. Vitamin $\mathrm{C}$ is an essential co-factor for collagen synthesis through the hydroxylation of proline and lysine and its deficiency leads to impaired collagen synthesis and poor wound healing [3]. Smoking induced impairment of proliferation and remodeling provides a good explanation of delayed healing, wound dehiscence and incisional hernia formation, which is seen significantly more frequently in smokers than in nonsmokers [3].

Borad, et al. studied effect of smoking on postoperative outcome in ventral hernia repair. The study included 32,973 smokers and 136,485 non-or ex-smoker patients who underwent ventral hernia repair. They found statistically significant increased incidence of 30 -day mortality $(0.27 \%$ vs $0.21 \%, p=0.04), 30$ day all morbidity $(7.17 \%$ vs $5 \%, \mathrm{p}<0.0001)$, wound morbidity $(4.57 \%$ vs $3 \%, \mathrm{p}<0.0001)$, respiratory $(0.7 \%$ vs $0.36 \%, \mathrm{p}<0.0001)$ and cardiac morbidity $(0.26 \%$ vs $0.21 \%, \mathrm{p}-=0.05)$ in smokers [1].

\section{Abstinence of Smoking and Its Effect on Wound Healing}

Two weeks after smoking cessation, platelet aggregability and dysfunction are reduced [7]. After four weeks, circulating endothelial progenitor cells are restored suggesting reduction in endothelial injury and dysfunction $[14,15]$. Generation of reactive oxygen radicals decreases and Level of Vitamin $\mathrm{C}$ increases 4 weeks after smoking cessation [3]. 4 weeks of abstinence significantly enhance inflammatory cell and macrophage migration in the wound cavity [16]. Oxidative bacterial killing mechanism of neutrophils and macrophages is restored after 4 weeks however reversal of neutrophil and monocytes' chemotaxis occur slowly [7]. Furthermore, 4 weeks of abstinence causes restoration of proteolytic enzymes secretion from neutrophils [17]. Abstinence from smoking gradually reverses the wound contraction seen in smokers [3]. The improvement of inflammatory cell function and host defense after smoking cessation provides a potential mechanism for reduced infectious complications after at least 4 


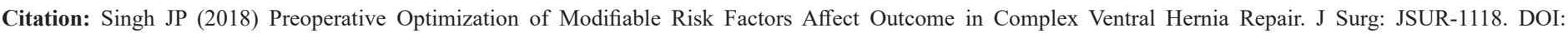
10.29011/2575-9760. 001118

weeks of abstinence [18]. Contrary to inflammatory cell function, wound proliferation and remodeling do not seem to reverse as fibroblast function, epidermal regeneration, collagen synthesis, and collagen degradation are unaffected by smoking cessation [18]. The lack of improvement in wound proliferation and remodeling may explain why abstinence does not reduce noninfectious healing complications such as necrosis, wound dehiscence and hernia [18].

\section{Nicotine Replacement Therapy and Wound Healing}

The adverse effects of nicotine replacement therapy for abstinent smokers on postoperative outcome are minimal and harmless [19]. Effects of pure nicotine on tissue oxygenation and microenvironment are transient and there is no evidence to suggest that repeated use of nicotine replacement drugs has a detrimental cumulative effect [3].

\section{Laboratory Test for Tobacco and Smoke Metabolites}

The most widely used biochemical marker of tobacco use is cotinine, the proximate metabolite of nicotine, which can be measured in blood, saliva, or urine [20]. Cotinine is quite specific for use of tobacco or nicotine-containing products. Cotinine has long half-life and its concentration does fluctuate greatly during the day. Blood level of cotinine is much higher than nicotine, thus facilitating its measurement. Cotinine would also be positive in patients on nicotine replacement therapy and therefore not a valid test for patients on replacement therapy [20]. Carbon monoxide (expired CO or carboxyhemoglobin) and thiocyanate may be used to detect heavy smoking but, being products of combustion, are not applicable for detecting smokeless tobacco use [20]. Anabasine and anatabine are present in tobacco but are not present in nicotine-containing medications and are not metabolically derived from nicotine $[20,21]$. Anabasine is present in trace amounts in tobacco smoke. Both anabasine and anatabine could be used for estimating tobacco consumption during nicotine replacement therapy [20,21].

\section{Electronic Cigarettes}

Clear evidence about the safety of e-cigarettes is lacking, and laboratory experiments and case reports suggest that these products may be associated with potential adverse health consequences. For now, E-cigarettes are not considered safe as nicotine replacement therapy [22].

\section{Recommendations}

- Smoking cessation for at least 4 weeks before complex hernia repair is recommended.

- Urine or serum cotinine level should be tested to confirm the abstinence from smoking.

- Nicotine replacement therapy is acceptable however cotinine test would be positive in these patients and therefore they should be tested for urine anabasine and anatabine level.

\section{Diabetes}

Association between hyperglycemia and susceptibility to infection is well established in diabetic patients [23]. Uncontrolled diabetes causes impairment of neutrophil adherence, chemotaxis, phagocytosis and intracellular bactericidal activity [24,25]. The degree of neutrophil impairment correlates with the degree of hyperglycemia [23]. Some of the defects can be partially reversed in vivo with intensive insulin treatment [26]. Hyperglycemia causes alterations in vascular permeability and normal redox reactions, which create a state of pseudohypoxia that further impairs the tissue defenses [27]. Glucose is a proinflammatory mediator that has been shown to have a wide variety of effects, including stimulating cytokine production and inhibiting endothelial nitric oxide levels. Even in healthy individuals, generation of reactive oxygen radicals by neutrophil and mononuclear cells is increased in the presence of high glucose, which adversely affects wound healing [28]. Dronge et al studied 490 diabetic patients and found that odds ratio of postsurgical infection in patients with poorly controlled diabetes $(\mathrm{HbA1c}>7)$ was $2.13(\mathrm{p}=0.07)$ [23]. Petro et al studied 306 patients with ventral hernia who underwent retromuscular repair and found poorly controlled diabetes to be an independent risk factor for postoperative wound morbidity. The study found that the odds ratio of wound complication was 2.41 $(p=0.01)$ in poorly controlled diabetic patients [29].

Stryker et al studied the effect of preoperative HbA1c and postoperative blood glucose on wound complications following total joint arthroplasty and identified that the odds ratio of wound complication was $3.75(\mathrm{p}=0.02)$ in patients with mean postoperative blood glucose of $>200 \mathrm{mg} / \mathrm{dl}$. The odds ratio of wound complication in patients with a maximum postoperative blood glucose of $>260 \mathrm{mg} / \mathrm{dl}$ was $3(\mathrm{p}=0.08)$. The odds ratio of wound complication in patients with preoperative HbAlc of $>6.7 \%$ was $9(p=0.03)$. This study identified the role of not only optimizing preoperative HbA1c but also a need to have a strict perioperative blood glucose control in order to decrease the postoperative wound complications [30]. Halkos et al studied the effect of preoperative HbAlc on sternal wound infection after coronary artery bypass surgery. It was found that patients with $\mathrm{HbA1c}>7 \%$ had 2.88 -fold increase risk of deep sternal wound infection compared with patients with $\mathrm{HbA} 1 \mathrm{c}<7 \%$. It was also found that with each unit increase in $\mathrm{HbA} 1 \mathrm{c}$, there was $31 \%$ increased risk of deep sternal wound infection [31].

Ramos et al studied the association of perioperative hyperglycemia and risk of postoperative infection in 995 patients who underwent various general and vascular procedures. It was found that with every 40-point increase in postoperative blood glucose above $110 \mathrm{mg} / \mathrm{dl}$, the postoperative infection rate increased 
Citation: Singh JP (2018) Preoperative Optimization of Modifiable Risk Factors Affect Outcome in Complex Ventral Hernia Repair. J Surg: JSUR-1118. DOI: $10.29011 / 2575-9760.001118$

by $30 \%[32]$.

\section{Recommended Interventions}

- Preoperative HbA1c level should be checked to assess the diabetes control.

- Patients with HbA1c level $>7.5$ should be referred to endocrinologist for better control of diabetes.

- Strict control of blood glucose should be practiced in the perioperative period.

\section{Preoperative Nutritional Supplementation}

Arginine is considered a non-essential amino acid and in the resting conditions arginine does not have any significant immuneenhancing role as very little arginine is utilized by myeloid or lymphoid cell lineages. Myeloid cell activation by surgery or trauma induces increased arginine uptake [33]. Inside the cells there is higher expression of Inducible Nitric Oxide Synthase (iNOS) and Arginase (ASE1). Arginine is then metabolized to nitric oxide by iNOS, which is necessary for normal immune function and killing of bacteria and parasites. ASE1 generates ornithine from arginine, which is important for generation of polyamines and proline, which are essential metabolites for a normal wound healing [33]. Arginine also plays important role in increased production of Interleukin-2, Interferon and tumor necrosis factor from helper $\mathrm{T}$ cells, which are required for wound healing [33]. Arginine is the precursor of growth factors like putrescine, spermine and spermidine. Via formation of glutamate, arginine yields increased amounts of proline and hydroxyproline, which are required for the synthesis of collagen. Clinical studies evaluating the effects of supplemental enteral arginine supply have demonstrated net nitrogen retention, increased protein synthesis and improved wound healing. Studies have also shown a clear benefit of arginine and omega 3 fatty acid supplementation not only in malnourished but also in well-nourished patients [34]. There is around 50\% reduction in infection rates in patients undergoing elective surgical procedures compared to controls $[33,35]$. The supplementation of arginine before surgery increases the arginine reserves and helps in preventing the deficiency of arginine due to activation of immune cells in the perioperative period. The supplementation of omega 3 fatty acids also helps in decreasing the depletion of arginine [34]. Commercially available immune enhancing products contain arginine and w- 3 fatty acids and usually prescribed for 5 days just before the surgery.

\section{Decolonization Protocols}

\section{Preoperative Showering with Chlorhexidine}

Preoperative showering with antiseptic agents has not shown to be beneficial in terms of decreasing wound complications in ventral hernia repair. Prabhu et al studied 3924 patients and identified two groups; 2,209 patients who received prehospital chlorhexidine bathing and 1,715 patients who did not receive prehospital bathing. The incidence of Surgical Site Infection (SSI) (OR 1.49, $\mathrm{p}<0.05$ ), Surgical Site Occurrence (SSO) (OR 1.34, $\mathrm{p}<0.05$ ) and SSO requiring procedural intervention (OR 1.07, $\mathrm{p}<0.05)$ was significantly higher in patients who received prehospital chlorhexidine bathing [36].

\section{Preoperative Bowel Preparation}

Krpata, et al. studied role of preoperative bowel preparation in ventral hernia repair. In class 1 cases, 313 patients were identified who received preoperative bowel preparation while 914 patients did not receive bowel preparation. It was found that patients who received preoperative bowel preparation had significantly higher SSI ( $4 \%$ vs $2 \%, p=0.04)$ and SSO (22 vs $14 \%, p=0.001)$. Surgical site occurrence requiring procedural intervention was also found to be higher in patients who received bowel preparation however it was not statistically significant ( $7 \%$ vs $5 \%, \mathrm{p}=0.10)$ [37].

\section{Staphylococcus aureus Eradication}

Staphylococcus aureus, including both methicillin-resistant and methicillin-susceptible strains, is the most common cause of surgical-site infection and ventilator-associated pneumonia and the second most common cause of central-catheter-associated bacteremia [38]. In community hospitals, Methicillin-Resistant Staphylococcus aureus (MRSA) has become the single most common cause of SSI [39]. The risk of health care associated infection is three to six times higher in nasal carriers of high-level Staphylococcus aureus compared to non-carriers or low-level carriers [40]. Ousley et al identified that history of MRSA infection regardless of body site, increases the surgical site infection rate in patients undergoing ventral hernia repair by 2.3 -fold $(p=0.035)$ [41]. Bebko, et al. demonstrated that preoperative MRSA decontamination in patients undergoing orthopedic hardware implantation decreased SSI rate by more than $50 \%(3.8 \%$ vs $1.1 \%, p=0.02$ ) [39]. Another randomized controlled trial showed that preoperative MRSA decolonization of positive nasal carriers with intranasal mupirocin and chlorhexidine bath had significantly lower Staph aureus infection than control group (3.4\% vs $7.7 \%$, p $<0.05)$ [40].

\section{Recommendations}

- All patients should be screened for MRSA by nasal swab preoperatively

- Positive carriers should be treated with $2 \%$ mupirocin ointment to be applied nasally twice daily along with $4 \%$ chlorhexidine bath for 5 days in the preoperative period.

- All patients with positive MRSA on screening should be given vancomycin in addition to cephalosporin as antibiotic prophylaxis. 


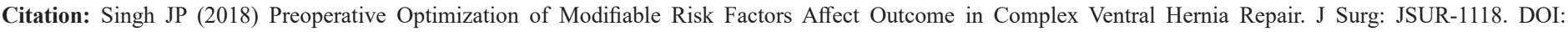
10.29011/2575-9760. 001118

- Routine preoperative chlorhexidine bathing and bowel preparation are not recommended in patients undergoing elective ventral hernia repair.

\section{Obesity}

Obese patients have higher incidence of surgical complications, including atelectasis, pneumonia, thrombophlebitis, wound dehiscence and infection, venous thromboembolism and mortality [42]. Adipose tissue has poor vascularity as capillary density does not increase proportionally to the increase in adipose tissue, which could potentially restrict capillary proliferation, leading to impaired angiogenesis [42]. The hypoperfusion of subcutaneous adipose tissue leads to decreased oxygen tension, which increases the risk of infection and necrosis. Excessive adiposity increases the expression of immature type III collagen and disorganized type I collagen causing impairment of proliferation and remodeling process of wound healing, which leads to higher risk of delayed healing, wound dehiscence and hernia formation in obese patients $[3,42]$. Sauerland et al identified that hernia recurrence increases with Body Mass Index (BMI) (risk ratio of 1.10 per unit BMI, $\mathrm{p}=0.01$ ) [43].

Study by Owei, et al. showed that patients with BMI $>40 \mathrm{~kg} /$ $\mathrm{m}^{2}$ had greater than twice the risk for operative and respiratory complications and the odds of these complications increased with increasing BMI [44]. The study concluded that strategies to encourage weight loss should be seriously considered prior to open ventral hernia repair, especially for patients with body mass index $>40 \mathrm{~kg} / \mathrm{m}^{2}$ [44]. Nelson et al studied 1,695 patients who underwent abdominal wall reconstruction between 2005 and 2010. 1,078 (63.2\%) patients were found to be obese (mean body mass index $=37.6 \mathrm{~kg} / \mathrm{m}^{2}$. Obese patients had significantly higher incidence of major surgical complications $(15.3 \%$ vs $10.1 \%$, $\mathrm{p}=.003)$, wound complications $(12.5 \%$ vs $8.1 \%, \mathrm{p}=.006)$ and return to operating room $(9.1 \%$ vs $5.4 \%, \mathrm{p}=.006)$ than non-obese patients. Obesity only directly led to a significantly increased odd of having a renal complication (OR 4.4, $\mathrm{p}=0.04)$ [45].

Another study by Giordano et al included 511 patients who underwent abdominal wall reconstruction with underlay mesh. Patients were divided into three groups on the basis of preoperative BMI: $<30 \mathrm{~kg} / \mathrm{m}^{2}$ (non-obese), 30 to $34.9 \mathrm{~kg} / \mathrm{m}^{2}$ (class I obesity), and $35 \mathrm{~kg} / \mathrm{m}^{2}$ or greater (class II/III obesity) [46]. They found that class I and class II/III patients had higher surgicalsite occurrence rates than non-obese patients $(26.4 \%$ vs $14.9 \%$, $\mathrm{p}=0.006 ;$ and $36.8 \%$ vs $14.9 \%, \mathrm{p}<0.001$, respectively) and higher overall complication rates $(37.9 \%$ vs $24.7 \%, \mathrm{p}=0.007$; and $43.4 \%$ vs $24.7 \%, \mathrm{p}<0.001$, respectively). Obese patients also had significantly higher skin dehiscence $(19.3 \%$ vs $7.2 \%, \mathrm{p}<0.001$; and $26.5 \%$ vs $7.2 \%, p<0.001$, respectively) and fat necrosis $(10 \%$ vs $2.1 \%, \mathrm{p}=0.001$; and $11.8 \%$ vs $2.1 \%, \mathrm{p}<0.001$, respectively) than non-obese patients. Patients with class II/III obesity had higher infection $(9.6 \%$ vs $4.3 \%, \mathrm{p}=0.041)$ and seroma rates $(8.1 \%$ vs $2.1 \%, p=0.006)$ than non-obese patients. Class I and class II/ III patients had higher hernia recurrence rates $(11.4 \%$ vs $7.7 \%, \mathrm{p}=$ 0.204 ; and $10.3 \%$ vs $7.7 \%, p=0.381$, respectively) [46]. A study on medical weight loss by Rosen et al concluded that utilization of a protein sparing modified diet in collaboration with a medical weight loss specialist is a valuable resource for guiding weight loss in patients with morbid obesity prior to elective complex surgical procedures [47]. Staged gastric bypass followed by ventral hernia repair was studied by Newcomb et al. 27 patients underwent ventral hernia repair after weight loss with gastric bypass at mean interval of 1.3 years. No recurrence was identified at an average follow up of 20 months [48].

\section{Recommendations}

- Elective ventral hernia surgery should be deferred until BMI 40 or less.

- Surgeons should have collaboration with medical weight loss specialist for modified protein sparing dieting program.

- Patients should be encouraged to participate in exercise plan along with modified protein sparing diet.

- Patients should be strongly considered for bariatric surgery for staged repair of ventral hernia if non-surgical weight loss programs are not effective.

\section{Conclusion}

Various factors have been identified, which could affect postoperative outcome after major surgical procedures. Smoking affects all phases of wound healing and increases risk of infection and hernia recurrence. Four weeks of abstinence reverses the impaired hemostatic and inflammatory phases and reduces the risk of infectious complications. Nicotine replacement therapy is acceptable as nicotine does not have similar adverse healing effects as smoking. Hyperglycemia also has adverse effects on wound healing and increases the risk of wound complications. Preoperative optimizations of diabetes along with strict perioperative glucose management have been found to decrease wound complications. Arginine is essential for normal wound healing and its level is decreased by surgical stress. Perioperative supplementation of arginine increases the arginine reserves and prevents its deficiency after surgery, which reduces the wound complications. Routine preoperative chlorhexidine bathing and bowel preparation have been associated with increased infectious complications and therefore not recommended. Nasal carriers of MRSA have been found to have higher risk of wound complications. Preoperative decontamination protocol with nasal mupirocin ointment and chlorhexidine bath has reduced incidence of infection. Obesity impairs the wound healing as well and has been associated with higher incidence of pneumonia, wound dehiscence and infection, 
Citation: Singh JP (2018) Preoperative Optimization of Modifiable Risk Factors Affect Outcome in Complex Ventral Hernia Repair. J Surg: JSUR-1118. DOI: $10.29011 / 2575-9760.001118$

venous thromboembolism and hernia recurrence. Weight loss is strongly recommended prior to elective hernia repair. Staged bariatric procedure followed by ventral hernia repair should be considered if exercise and medical weight loss programs are not effective.

\section{References}

1. Borad NP and Merchant AM (2017) The effect of smoking on surgical outcomes in ventral hernia repair: a propensity score matched analysis of the National Surgical Quality Improvement Program data. Hernia 21: $855-867$

2. Guo S and DiPietro LA (2010) Factors Affecting Wound Healing. J Dent Res 89: 219-229.

3. Sorensen LT (2012) Wound Healing and Infection in Surgery: The Pathophysiological ImpactofSmoking, Smoking Cessation, and Nicotine Replacement Therapy A Systematic Review. Ann Surg 255: 1069-1079.

4. Sorensen LT, Jorgensen S, Petersen LJ, Hemmingsen U, Bülow J, et al. (2009) Acute effects of nicotine and smoking on blood flow, tissue oxygen, and aerobe metabolism of the skin and subcutis. J Surg Res 152: $224-230$.

5. Black CE, Huang N, Neligan PC, Levine RH, Lipa JE, et al. (2001) Effect of nicotine on vasoconstrictor and vasodilator responses in human skin vasculature. Am J Physiol Regul Integr Comp Physiol 281: R1097-R1104.

6. Jensen JA, Goodson WH, Williams H, Hunt TK (1991) Cigarette smoking decreases tissue oxygen. Arch Surg 126: 1131-1134.

7. Sorensen LT, Nielsen HB, Kharazmi A, Gottrup F (2004) Effect of smoking and abstention on oxidative burst and reactivity of neutrophils and monocytes. Surgery 136: 1047-1053.

8. Zappacosta B, Martorana GE, Papini S, Gervasoni J, lavarone F, et al (2011) Morpho-functional modifications of human neutrophils induced by aqueous cigarette smoke extract: comparison with chemiluminescence activity. Luminescence 26: 331-335.

9. Stringer KA, Tobias M, O'Neill HC, Franklin CC (2007) Cigarette smoke extract-induced suppression of caspase-3-like activity impairs human neutrophil phagocytosis. Am J Physiol Lung Cell Mol Physiol 292: L1572-L1579.

10. Bridges RB and Hsieh L (1986) Effects of cigarette smoke fractions on the chemotaxis of polymorphonuclear leukocytes. J Leukoc Biol 40: 73-85.

11. Nagy J, Demaster EG, Wittmann I, Shultz P, Raij L (1997) Induction of endothelial cell injury by cigarette smoke. Endothelium 5: 251-263.

12. Mohsenin V and Gee JL (1989) Oxidation of alpha 1-protease inhibitor: role of lipid peroxidation products. J Appl Physiol 66: 2211-2215.

13. Gottrup F (2004) Oxygen in Wound Healing and Infection. World J Surg 28: 312-315.

14. Kondo T, Hayashi M, Takeshita K, Numaguchi Y, Kobayashi K, et al (2004) Smoking cessation rapidly increases circulating progenitor cells in peripheral blood in chronic smokers. Arterioscler Thromb Vasc Biol 24: 1442-1447.

15. Moreno H Jr, Chalon S, Urae A, Tangphao O, Abiose AK, et al. (1998) Endothelial dysfunction in human hand veins is rapidly reversible after smoking cessation. Am J Physiol 275: H1040-H1045.
16. Sorensen LT, Toft B, Rygaard J, Ladelund S, Teisner B, et al. (2010) Smoking attenuates wound inflammation and proliferation while smoking cessation restores inflammation but not proliferation. Wound Repair Regen 18: 186-192.

17. Sorensen LT, Zillmer R, Agren M, Ladelund S, Karlsmark T, et al. (2009) Effect of smoking, abstention, and nicotine patch on epidermal healing and collagenase in skin transudate. Wound Repair Regen 17: 347-353.

18. Sorensen LT, Karlsmark T, Gottrup F (2003) Abstinence from smoking reduces incisional wound infection: a randomized controlled trial. Ann Surg 238: 1-5

19. Warner DO (2006) Perioperative abstinence from cigarettes: physiologic and clinical consequences. Anesthesiology 104: 356-367.

20. Jacob P, Hatsukami D, Severson H, Hall S, Yu L, et al. (2002) Anabasine and anatabine as biomarkers for tobacco use during nicotine replacement therapy. Cancer Epidemiol Biomarkers Prev 11: 1668-1673.

21. Jacob P, Yu L, Shulgin AT, Benowitz NL (1999) Minor tobacco alkaloids as biomarkers for tobacco use: comparison of users of cigarettes, smokeless tobacco, cigars, and pipes. Am J Public Health 89: 731-736.

22. Ebbert JO, Agunwamba AA, Rutten LJ (2015) Counseling patients on the use of electronic cigarettes. Mayo Clin Proc 90: 128-134.

23. Dronge AS, Perkal MF, Kancir S, Concato J, Aslan M, et al. (2006) Long-term glycemic control and postoperative infectious complications. Arch Surg 141: 375-380.

24. Geerlings SE and Hoepelman Al (1999) Immune dysfunction in patients with diabetes mellitus (DM). FEMS Immunol Med Microbiol 26: 259-265.

25. Delamaire $\mathrm{M}$, Maugendre $\mathrm{R}$, Moreno $\mathrm{M}$, Goff $\mathrm{MC}$, Allannic $\mathrm{H}$, et al. (1997) Impaired leucocyte functions in diabetic patients. Diabet Med 14: 29-34.

26. Rassias AJ, Marrin CA, Arruda J, Whalen PK, Beach M, et al. (1999) Insulin infusion improves neutrophil function in diabetic cardiac surgery patients. Anesth Analg 88: 1011-1016.

27. Williamson JR, Chang K, Frangos M, Hasan KS, Ido Y, et al. (1993) Hyperglycemia pseudohypoxia and diabetic complications. Diabetes 42: 801-825.

28. Marik PE and Raghavan M (2004) Stress-hyperglycemia, insulin and immunomodulation in sepsis. Intensive Care Med 30: 748-756.

29. Petro CC, Posielski NM, Raigani S, Criss CN, Orenstein SB, et al. (2015) Risk factors for wound morbidity after open retromuscular (sublay) hernia repair. Surgery 158:1658-1668.

30. Stryker LS1, Abdel MP, Morrey ME, Morrow MM, Kor DJ, et al. (2013) Elevated postoperative blood glucose and preoperative hemoglobin A1C are associated with increased wound complications following total joint arthroplasty. J Bone Joint Surg Am 95: 808-814.

31. Halkos ME, Thourani VH, Lattouf OM, Kilgo P, Guyton RA, et al. (2008) Preoperative Hemoglobin A1c Predicts Sternal Wound Infection After Coronary Artery Bypass Surgery with Bilateral Versus Single Internal Thoracic Artery Grafts. Innovations (Phila) 3: 131-138.

32. Ramos M, Khalpey Z, Lipsitz S, Steinberg J, Panizales MT, et al. (2008) Relationship of perioperative hyperglycemia and postoperative infections in patients who undergo general and vascular surgery. Ann Surg 248: 585-591. 
Citation: Singh JP (2018) Preoperative Optimization of Modifiable Risk Factors Affect Outcome in Complex Ventral Hernia Repair. J Surg: JSUR-1118. DOI: $10.29011 / 2575-9760.001118$

33. Ochoa JB, Makarenkova V, Bansal V (2004) A rational use of immune enhancing diets: when should we use dietary arginine supplementation? Nutr Clin Pract 19: 216-225.

34. Braga M (2012) Perioperative immunonutrition and gut function. Curr Opin Clin Nutr Metab Care 15: 485-488.

35. Suchner U, Heyland DK, Peter K (2002) Immune-modulatory actions of arginine in the critically ill. Br J Nutr 87: S121-132.

36. Prabhu AS, Krpata DM, Phillips S, Huang LC, Haskins IN, et al (2017) Preoperative Chlorhexidine Gluconate Use Can Increase Risk for Surgical Site Infections after Ventral Hernia Repair. J Am Coll Surg 224: 334-340.

37. Krpata DM, Haskins IN, Phillips S, Prabhu AS, Rosenblatt S, et al. (2017) Does Preoperative Bowel Preparation Reduce Surgical Site Infections During Elective Ventral Hernia Repair? J Am Coll Surg 224: 204-211.

38. Huang SS, Septimus E, Kleinman K, Moody J, Hickok J, et al. (2013) Targeted versus universal decolonization to prevent ICU infection. $\mathrm{N}$ Engl J Med 368: 2255-2265.

39. Bebko SP, Green DM, Awad SS (2015) Effect of a preoperative decontamination protocol on surgical site infections in patients undergoing elective orthopedic surgery with hardware implantation. JAMA Surg 150: 390-395.

40. Bode LG, Kluytmans JA, Wertheim HF, Bogaers D, VandenbrouckeGrauls CM, et al. (2010) Preventing surgical-site infections in nasal carriers of Staphylococcus aureus. N Engl J Med 362: 9-17.

41. Ousley J, Baucom RB, Stewart MK, Phillips SE, Holzman MD, et al. (2015) Previous Methicillin-Resistant Staphylococcus aureus Infection
Independent of Body Site Increases Odds of Surgical Site Infection after Ventral Hernia Repair. J Am Coll Surg 221: 470-477.

42. Pierpont YN, Dinh TP, Salas RE, Johnson EL, Wright TG, et al. (2014) Obesity and Surgical Wound Healing: A Current Review. ISRN Obes 2014: 638936.

43. Sauerland S, Korenkov M, Kleinen T, Arndt M, Paul A (2004) Obesity is a risk factor for recurrence after incisional hernia repair. Hernia 8: 42-46.

44. Owei L, Swendiman RA, Kelz RR, Dempsey DT, Dumon KR (2017) Impact of body mass index on open ventral hernia repair: A retrospective review. Surgery 162: 1320-1329.

45. Nelson JA, Fischer JP, Cleveland EC, Wink JD, Serletti JM, et al. (2014) Abdominal wall reconstruction in the obese: an assessment of complications from the National Surgical Quality Improvement Program datasets. Am J Surg 207: 467-475.

46. Giordano SA, Garvey PB, Baumann DP, Liu J, Butler CE (2017) The Impact of Body Mass Index on Abdominal Wall Reconstruction Outcomes: A Comparative Study. Plast Reconstr Surg 139: 1234-1244.

47. Rosen MJ, Aydogdu K, rafmiller K, Petro CC, Faiman H, et al. (2015) A Multidisciplinary Approach to Medical Weight Loss Prior to Complex Abdominal Wall Reconstruction: Is it Feasible J Gastrointest Surg 19: 1399-1406.

48. Newcomb WL, Polhill JL, Chen A, Kuwada TS, ersin KS, et al. (2008) Staged hernia repair preceded by gastric bypass for the treatment of morbidly obese patients with complex ventral hernias. Hernia 12: 465-469. 